\title{
(2) OPEN ACCESS \\ Aligning quality improvement with better child health for the 21st century
}

\author{
Guddi Singh (10, 1,2 Alan Cribb ${ }^{2}$
}

\begin{abstract}
'Mary Sheridan Centre for Child Health, Guy's and St Thomas' Hospitals NHS Trust, London, UK ${ }^{2}$ Faculty of Social Science and Public Policy, King's College London, London, UK
\end{abstract}

\section{Correspondence to}

Professor Alan Cribb, Centre for Public Policy Research, King's College London, London, UK; alan.cribb@kcl.ac.uk

Received 25 January 2020 Revised 23 October 2020 Accepted 10 November 2020

\section{Check for updates}

(c) Author(s) (or their employer(s)) 2020. Re-use permitted under CC BY. Published by BMJ.

To cite: Singh G, Cribb A. Arch Dis Child Educ

Pract Ed Epub ahead of print: [please include Day Month Year]. doi:10.1136/ archdischild-2020-318924

\section{ABSTRACT}

Quality improvement (QI) has tremendous potential to tackle the shortcomings of health services. But health professionals have not yet fully embraced QI as part of their day-to-day concerns. Indeed, QI is sometimes experienced as a brake on quality rather than a catalyst for improvement. This can happen, for example, if there is too much emphasis on meeting shortterm institutional goals rather than on addressing long-term health needs. This emphasis also risks equating quality with safety and efficiency measures while neglecting patient-centredness and equity. QI does not have to be like this. We suggest that the conscientious and critical engagement of health professionals in QI can lead to genuinely better and more far-reaching outcomes for child health. We also distinguish between QI projects that repair the status quo and those that seek to reform it, arguing that there is an important place for both.

\section{INTRODUCTION}

The UK's Care Quality Commission (CQC) - the regulatory body tasked with regulating and inspecting health and social care services-recently reported that it is those National Health Service (NHS) Trusts that have successfully embedded a culture of quality improvement (QI) and continuous improvement that are delivering the highest quality care. ${ }^{1}$ The inference is that all healthcare providers ought to be adopting a QI focus in order to meet quality standards. But is QI really the answer?

We contend that QI ought to be enthusiastically embraced but also, like all innovations or developments in healthcare, met with what we will call 'critical engagement'. Otherwise, the danger is that the way that QI is sometimes interpreted within health systems and foisted on healthcare professionals can actually act as a brake on quality rather than a catalyst.

Why is this? And how can we make the most of QI's potential in healthcare delivery? We explore these questions with reference to child health in the UK and use examples to illustrate how different ways of approaching QI can be aimed at very different results.

We argue that for QI not to act as a brake, it needs to be more deeply rooted in what matters in people's lives-in the lives of children and families and in professional vocations. We suggest that a more socially and professionally engaged approach to QI is key to adopting the most appropriate strategies. Finally, we suggest that realigning quality improvement so that it is directed towards what matters most in child health should lead to more ambitious improvement agendas that interpret quality as about reform, and not only 'repair', of the health system.

\section{UNREALISED POTENTIAL OF QI}

QI has tremendous potential to tackle the shortcomings of health services including the harms they can cause. What is more, QI is now accepted as a major policy priority in the NHS and an important plank of healthcare professionalism. QI offers particular promise to child health in the UK, which is rife with evidence of wide variations in the quality of care, poorly coordinated care, wasted resources and iatrogenic harm. ${ }^{2}$ The need for existing child health systems to evolve to better meet current and future demands effectively is clear and uncontroversial.

The launch of the Royal College of Paediatrics \& Child Health Quality Improvement Training Programme along with support from the Institute for Healthcare Improvement and the now defunct National Institute for Innovation and Improvement-on the back of 


\section{Box 1 Repair models}

\section{Example 1: Electronic growth charts}

- An example is the introduction of electronic growth charts at one hospital trust. ${ }^{41}$ Accurate evaluation of growth is a key assessment of child health, and yet current paper-based practices are unreliable and inaccurate. The generation of electronic growth charts of every child seen at one hospital trust has resulted in impressive increases in documentation and shareability of data. This is an important dimension of success.

\section{Analysis: Limited ambition, limited impact}

- However, it can be seen as limited. Moreover, health professionals who were critically engaged might ask: to what end? Such projects are successful in that they produce clear 'improvements' in a narrow range of impact measures, but what is not clear is the extent to which outcomes that matter to health professionals and patients have improved. What would the benefit of more accurate measurements be if the weights measured did not reveal the complexity of the kinds of malnutrition in today's children? Deeper questions about why more accurate documentation matters or whether such interventions are related to better health outcomes in children can sometimes be elided in preference for the winnable, measurable and fixable.

- In addition, the effects of these kinds of projects, while clearly an improvement on previous baseline measures, are often short-lived. More insidiously, by their very success, they can shift the problem to another part of the system.

\section{Example 2: The 4-hour wait in A\&E}

- Since $2005,98 \%$ of patients treated in England's emergency departments (EDs) must be discharged or placed in a hospital bed within 4 hours of arrival, including children and young people. By imposing fines on hospitals where this 4-hour target has been breached, there is pressure on health professionals and care systems to process patients through the ED as quickly as possible. While not explicitly a 'QI initiative', the aim was to improve the quality of hospital care by addressing severe ED crowding, and the target since became a key performance standard for Acute NHS Hospital Trusts.

\section{Analysis: Meeting targets, missing the point}

- But while patients may well be seen more quickly in the $E D$, the effect of its unintended consequences and perverse incentives is delay and danger in other parts of the hospital. Although most EDs are now within range of the target, consistent performance while balancing patient safety - particularly in paediatrics — remains tenuous. ${ }^{42}{ }^{43}$ For example, the target is blind to how long patients wait once the ED standard is missed. This does not mean that ED staff simply 'give up' on delivering timely care for patients who have breached the 4-hour standard, but it does mean that the waiting times for these patients are obscured from the key national figures. These problems have led to current proposals to replace the 4 -hour target. ${ }^{44}$ Such initiatives mask the real,

\section{Box 1 Continued}

underlying problems, such as insufficient or unevenly distributed resources, which are often complex in nature. In this way, such projects can have the perverse effect of being an obstacle to bigger change. By inhibiting the recognition and addressing of quality more broadly, such QI has unintended negative side effects.

hospital-led initiatives like EQUIP at Great Ormond Street Hospital in London ${ }^{3}$ - provides a real opportunity for paediatrics to be the first specialty to embrace QI as a core part of its professional responsibilities and to provide a new generation with the right skills to turn this potential into reality. But there is a long way to go. In the UK, junior doctors' attitudes towards QI are mixed: while there is agreement on the importance of QI in terms of patient safety, not all share enthusiasm for engaging in QI. ${ }^{4}$ Comparable studies in the US focusing on paediatrics have revealed that although national bodies have proclaimed the importance of incorporating QI into the daily fabric of clinical care, the evidence of practicing paediatricians participating in QI activities is much thinner. ${ }^{5}$ It seems that health professionals, including doctors, are yet to be fully convinced of the virtues and value of QI.

We would suggest that caution about, or even resistance to, some forms of QI may be justified while, at the same time, arguing for the importance of professional engagement with and ownership of an expanded QI remit. The success of QI approaches to improve diabetes control, ${ }^{6}$ reduce inpatient length of stay ${ }^{7}$ and increase the safety of life-saving procedures in paediatrics, ${ }^{8}$ mean that not playing the game is not an option; the potential gains and benefits of QI to child health are too valuable a prize to miss.

We suggest, instead, that health professionals ought to conscientiously engage in QI activities but that this requires scepticism as well as commitment. We are not arguing for blanket scepticism, let alone cynicism, but a whole-hearted commitment to healthcare quality that embraces the possibilities of QI while being critically reflective about the strengths and weaknesses of specific QI initiatives. This involves drawing on and exercising the full range of their professional visions, capabilities and ethics, and attending to the purpose of the health systems they operate in, in order to ensure that QI initiatives have the most potential to do good.

To appreciate what such a re-imagined QI could be, we first need to highlight how QI is currently commonly interpreted in many practical child health contexts, the limitations of this interpretation, and why this leads to some instances of QI not only being ineffective but acting as a brake on quality in child health. 


\section{Box 2 Reform models}

\section{Example: Children and Young People's Improvement} Collaborative (CYPIC)

- The Children and Young People's Improvement Collaborative (CYPIC) in Scotland took a commonly experienced problem — poor child health outcomesand started asking different questions. If the purpose of health systems is to help everyone in society to be healthier then, what does 'good' look like? No less grounded in quantitative data around public health outcomes and causes of illness, CYPIC reasoned that achieving more equitable improvements in health needed to come through addressing the most influential determinants of health-the social factors causing illness and disease. By applying QI throughout the child's journey from pre-birth to adulthood, this national programme ambitiously aims to interrupt the cycle of deprivation and associated impact on child health outcomes by making 'Scotland the best place to grow up'.

- This focus entails QI efforts that extended beyond the traditional boundaries of what is considered the health system to empower workforce practitioners to improve the delivery of care across sectors as varied as schools, prisons and the home. Examples of some of their initiatives include school-based projects to develop social and emotional resilience and increase childhood numeracy, helping fathers in prison to understand their children's needs and build positive family relationships, and integrated financial and literacy support for pregnant women on low incomes to increase income by up to f5000 per family and ensured access to Healthy Start Vouchers so that pregnant women and children get the nutrition they need.

\section{Analysis: Asking what really matters}

- CYPIC is explicit about using QI as a low-risk, low-cost method to tackle inequality and build equity in Scotland. Moving beyond waiting times or patients seen, measures of success with such an approach are necessarily expansive and include raising livings standards for Scotland's poorest families, improved school attainment and reduced criminality across the population. Still a work in progress, the CYPIC system has been showcased and presented around the world as an exemplar of QI that has resulted in potentially long-standing benefits in outcomes that matter to the people served by and working in the system. ${ }^{45}$

\section{PREVAILING CONCEPTIONS OF QI IN CHILD HEALTH}

Within the English NHS, as in many places, there is a tendency for prevailing conceptions of quality to be narrow, technical and 'defensive', rather than broader and more aspirational. This can lead to a culture stifling to improvement. Our argument is that when QI is associated with narrow approaches to quality, then, this not only limits the potential for what might be improved and how, but also limits who will get

\section{Box 3 Stepping-stone models}

\section{Example: Addressing child poverty in clinical practice}

- Trainee paediatricians in a London district general hospital used QI methodology in the emergency department to develop clinical surveillance tools and advice for addressing poverty in practice. ${ }^{46}$ Simple educational interventions and plan-do-study-act (PDSA) cycles to create and refine both screening tools and resource materials succeeded in ensuring stakeholder buy-in along with a substantial increase in the identification of child poverty and awareness of local resources over just a few months. The project is on-going and developing iteratively, but already there are indications that both staff and patients are finding this approach helpful, with reduced feelings of isolation and increased levels of knowledge and feelings of empowerment. Given that non-urgent use of the emergency department is linked to socioeconomic stress, psychiatric comorbidities and a lack of social support, ${ }^{47}$ it may well be that this small QI project could ultimately lead to reduced emergency service admissions.

\section{Analysis: Tapping into intrinsic motivations and changing cultures}

- Importantly, the project was not motivated by a need to see more patients more quickly or to improve patient safety; rather it arose from a desire to improve healthcare professionals' ability to address larger societal needs and injustices and to tackle the problems that matter to patients. Though small in scale, the project explicitly set out to change the culture of the working environment and to catalyse change elsewhere. Adaptation of the original project at another hospital site has resulted in bigger conversations about the purpose of local healthcare services, with the possibility of new clinical roles and pathways being posited. Originating in a broader conception of quality, then, the effects have been wide-ranging and are likely to be more sustainable, all while potentially reducing admissions to hospital and reducing the burden on acute services.

involved. Instead, we suggest that QI needs to grapple with what really matters to people's lives - both service users and professionals. Otherwise it risks losing people who may be put off by the tools (QI methods), which have come to stand for the whole (quality care).

The prevailing narrow conception of quality in health arguably comes from two directions. First, the shift towards more managerial, marketised and fragmented healthcare delivery in the NHS in recent years has meant an ever-increasing focus on meeting budget pressures and institutional targets. Second, some of the narrowness of QI is left over from the origins of the field itself; although, as we will go on to stress, the field has evolved substantially since its origins. In asking how to improve and transform healthcare, the pioneers of QI based improvement models on those from the manufacture of products ranging from 


\section{Box 4 Summary}

- Quality improvement (QI) has tremendous potential to tackle the shortcomings of health services. But health professionals have not yet fully embraced QI as part of their day-to-day concerns. QI is often experienced as a brake on quality rather than a catalyst.

- Prevailing conceptions of quality improvement are unbalanced. There is too much emphasis on meeting short-term institutional goals rather than on addressing long-term health needs. This also often means equating quality with safety and efficiency measures while neglecting patient-centredness and equity.

- QI does not have to be like this. We suggest that the conscientious and critical engagement of health professionals in QI can lead to genuinely better and more far-reaching outcomes for child health.

- We also distinguish between QI projects that repair the status quo and those that seek to reform it, arguing that there is an important place for both.

electronics to cars. Healthcare organisations were conceived of as factory production lines and human bodies as 'widgets'. The assumption was that 'making a product' and 'making a service' were similar-they were both systems for 'making'. ${ }^{9}$ This heavily 'technicist' emphasis suited biomedicine's existing tendency towards reductionism and measurement. It also reinforced the idea that innovation in healthcare is linear rather than networked or emergent. ${ }^{10}$ This has led, at least until recently, to a QI which often treats improvement as about 'fixing' service shortcomings by locally designing and implementing interventions using a repertoire of 'QI methods'. ${ }^{11} 12$ When this happens,
QI, as method, has effectively become dislodged from and bears only superficial relation to quality as theory, which is rooted in a rich and ever expanding scholarly literature of improvement science. ${ }^{13-18}$

In practice this means that a limited 'mind the gap' mentality is often favoured over one that embraces openness and complexity. ${ }^{19}$ This results in a narrow, quantitative focus on fixing 'fixable' problems with silver bullets, ${ }^{20}$ and discourages the harder thinking and reorganising that more radical, deepseated change would entail. ${ }^{21}$ Even high-profile and successful approaches to QI may suffer from defining quality in restricted ways. An illustrative example is the "No Waste, No Waits, Zero Harm" initiative at Great Ormond Street, where the Quality and Safety Team's job is to support the provision of "a safe, effective and efficient service" to patients and families. But what of the other Institute of Medicine's 'dimensions' of quality? In the NHS there tends to be a high level of stress on both 'efficiency' and 'safety' (and related concerns including 'effectiveness' and 'timeliness') compared with the dimensions of 'equity' and 'patientcentredness'. which are all too often incorporated more as a welcome side-effects rather than as primary goals worthy of pursuit in themselves. While the Great Ormond Street initiative did make efforts to place patients and carers at the heart of change, the branding of the project focused on addressing delays and safety, thereby implying, albeit unintentionally, what the motivating goals of the project were. By contrast, examples from outside the NHS including those of Dartmouth Microsystem and Cincinnati Children's Hospitals in the United States are held up as paragons of QI excellence precisely because they explicitly and

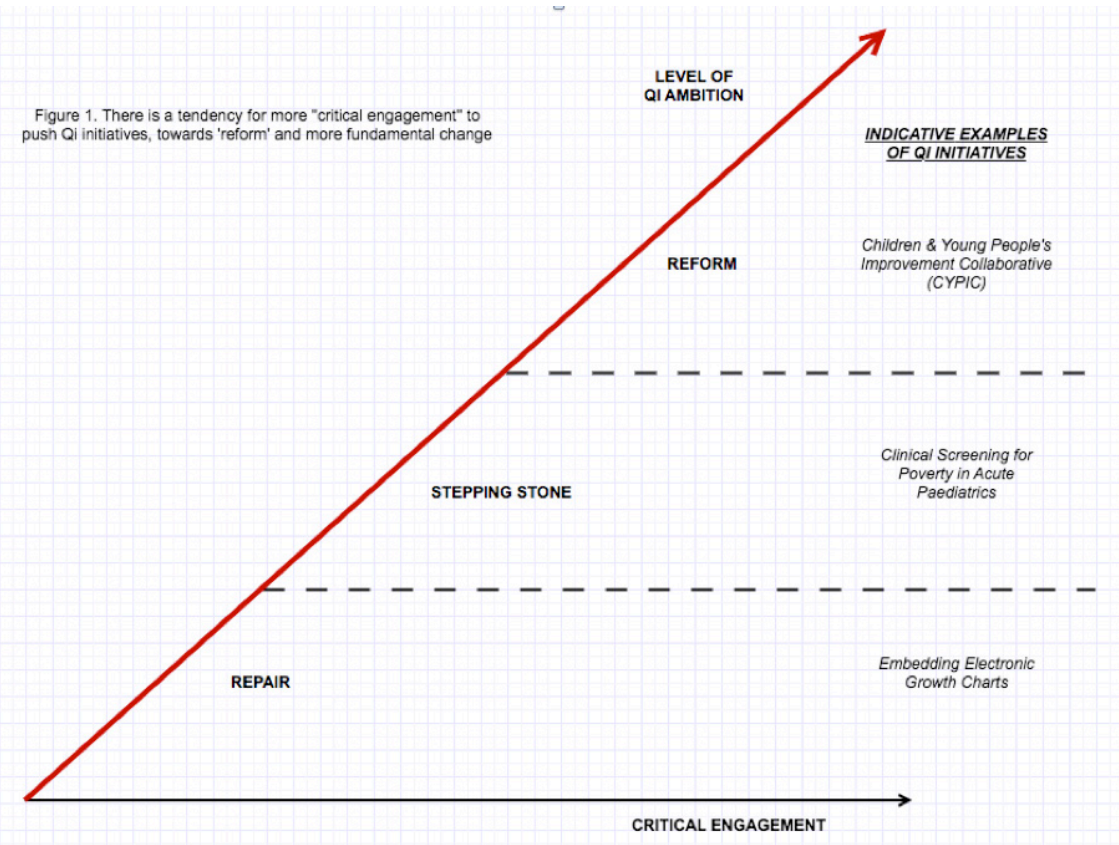

Figure 1 There is a tendency for more 'critical engagement' to push QI initiatives from 'repair' to 'reform' and more fundamental change. 
successfully target all six of the IoM's dimensions of quality. These are important considerations for the UK when inequalities and inequity in service provision loom large ${ }^{22}$ and patient-centredness remains poorly executed. ${ }^{23}$ No one could reasonably argue that efficiency and safety are unimportant. However, there is a danger that the institutional stress on these important concerns-often understood in relatively narrow and short-term ways-may result in them being treated as roughly synonymous with quality, thereby potentially obscuring other dimensions and conceptions of quality.

The Care Quality Commission inspections are a prime example of the potentially distorting effects of broader quality discourses. These annual visits—ostensibly in service of raising quality standards-produce a frenzy of activity that make sense from an institutional perspective but which are more difficult to defend from professional perspectives. The point of these activities is, in many respects, the demonstration of achievement around quality indicators and narratives rather than achievement of good quality. For example, increased care will be taken to check that data are 'presentable', that diagnoses are correctly coded, that targets have been met, that walls are nicely decorated and that wards seem 'welcoming'. Once again, none of these things are unimportant, but for busy professionals they can result in a sense that attention is being placed on the wrong things, and sometimes in what feels like arbitrary ways. This sense of misplaced focus or arbitrariness is reinforced by the judgement that some of the things being prioritised do not contribute to 'better care' for patients or clinicians in any meaningful way. At the margins, these practices can give 'quality' a bad name-'quality' becomes something that gets in the way of doing a good job rather than a helpful stimulus for working towards imagining and realising better healthcare. These practices also have the potential to undermine the vocational identities and commitments of professionals. Most doctors, for instance, see providing high-quality care as a professional responsibility and also as their raison d'être. ${ }^{24} 25$ But if they come to associate the institutional interpretation of 'quality' with something that gets in the way of their vocation that is counter-productive from everyone's point of view, it may contribute to professional cynicism and also thereby indirectly undermine quality and sustainability. A survey among GPs indicated that $80 \%$ of doctors saw CQC inspections as involving excessive preparation, taking doctors away from patients and thereby contributing to professional stress, and this at a time when there is already considerable concern about staff recruitment, retention and well-being. ${ }^{26}$

One potentially useful way of responding to these concerns would be to differentiate between broader processes of 'quality management' or 'quality assurance' on the one hand and 'quality improvement' more specifically-where the latter is seen as a specialism that applies improvement science. QI in this specialist sense would typically recommend, and be rooted in, the continuous involvement of professionals. Furthermore it would not divorce methods from theory, and would counsel against 'projectitis ${ }^{20}$ — that is the problems caused by pursuing disconnected projects and activities rather than a more systems-based approach to strategic improvement. We are happy to endorse these arguments that defend specialist quality improvement from looser uses of the QI language. But here we are deliberately questioning the broader range of 'real world' ways that quality discourses and practices shape the field of child health. In addition we want to highlight the way that the knowledge base of improvement can be yet further enlarged and extended ${ }^{27}$-a process that is well underway-so as to support more ambitious and reforming quality agendas.

\section{Richer conceptions of, and approaches to, QI}

Of course not all QI is a brake on quality. Nor is it all 'distorted' by managerial or other conflicting norms and pressures. There are plentiful examples of QI within healthcare with high levels of staff and serviceuser buy-in and participation and with successful outcomes. Indeed, to cast QI in a negative light as simply 'technicist' would be a completely unfair reflection of what has always been a much more complex and promising field of activity. Influential figures have long advocated for the need for imagination and flexibility in $\mathrm{QI}^{28}{ }^{29}$ - casting QI as 'art' as both a science and an 'art'. Also, current leaders within improvement science have called for QI to embrace even broader perspectives, epistemologies and methods. This includes, for example, (1) placing an equal emphasis on strengthening 'contexts' 30 as well as on making 'interventions' (and on understanding the relationships between these things) ${ }^{31}$; (2) more use of sociological lenses-including, but not limited to, attention to the social determinants of health and the organisational and cultural landscapes of improvement ${ }^{32}$; (3) the role of co-production and the scope for co-design in $\mathrm{QI}^{33} 34$ and-following from all these-(4) the central importance of understanding and harnessing the values of all stakeholders including patients, professionals and relevant populations. As well as anchoring QI in what matters to people in the long run rather than shortterm institutional targets, this allows room for broader social and radical agendas as reflected in the rise of the population health sciences movement. ${ }^{35}$ This broader conception of QI also allows for a more positive conception of health (an interest in flourishing as well as the absence of pathology), is open to the reimagining of healthcare roles and boundaries, and has led to ideas such as equity and patient-centeredness taking more prominent positions in QI discourses. ${ }^{36-39}$

How can QI efforts be conducted in ways that matter to both service users and health professionals so as to have the best chance to do good? 
We argue that for the field of QI in healthcare to fulfil its potential, what is required is conscientious professional engagement with the field. This means drawing on the expanding field of improvement science, including the explicit and careful consideration of sociological and philosophical perspectives on improvement-which, for short, we are calling 'critical engagement'. In essence, the more thought that has gone into, and the more attention that has been paid to, the values, purposes and methods of QI efforts, the more likely it is that quality will genuinely be improved in meaningful ways. As we have noted improvement scientists are sceptical about QI that is too piecemeal (that suffers from 'projectitis') rather than an approach that is a more systems-oriented and sustainable. ${ }^{12} 16$ We would argue that critical engagement with QI on the part of professionals will strongly reinforce the importance of taking this broader perspective.

Boxes 1-3 use real-life examples to illustrate 'good QI' initiatives in child health along a spectrum from those that repair the status quo to those that seek to reform it (figure 1). We wish to stress that initiatives all along this spectrum can be worthwhile and provide examples of good practice. However, we would note that the distinction between narrowly focussed approaches to quality and the moretheoretically informed and critically engaged approaches that we have been advocatinghas implications for the scope and ambition of QI. It pushes QI thinking away from short-term institutional goals and towards greater ambition, more fundamental agendas and thereby towards the 'reform' end of the spectrum.

\section{Repair models: change in the here and now}

Projects that enhance the status quo are common. The aim of the approach is to repair what already exists. They are easiest, although not easy, to implement: As discrete, time-limited, small-scale projects, they require minimal disruption or reorganisation to existing services and systems, and they provide immediate or rapid gratification by improving selected measures in the here and now (box 1).

\section{Reform models: transforming the future}

Projects that seek to be more transformative are much less common, owing to the greater investment in time, money and resources required to bring about this kind of change. These initiatives reconfigure services and upend existing systems. Such wholesale reform, as opposed to repair, comes from its creators asking difficult questions about the intended purpose of the health system, the functions to be served, to whom and by whom, and the outcomes that matter (box 2).

\section{Stepping-stone models: bridges to transformation}

Though we have drawn the contrast between improvement work that broadly maintains the status quo on the one hand, and that which is transformative on the other, good QI is not restricted to these two extremes. It is entirely possible for QI to have transformative potential without taking the form of large-scale service reconfiguration. Indeed, initiatives may be small in terms of size and rely on the limited palette of existing QI methods, and yet potentially represent a substantial change of emphasis by virtue of being oriented towards ends beyond efficiency and safety (box 3 ).

\section{CONCLUSION}

QI has great potential for improving healthcare delivery and health and well being in society; however, we suggest its impact will be greatest when it is part of a coherent, organisation-wide approach ${ }^{40}$ and also practised with thoughtful deliberation about the purposes and values that drive these initiatives. If we want to provide future paediatricians with a solid grounding in QI, it must include, but also move beyond, the quality conceptions and tools most readily to hand. Indeed, we should insist that more explicit, broad-ranging and honest conversations about the nature of quality and the possibilities and complexities of QI are had before pressing ahead with more and more QI initiatives. This would entail open-minded philosophical and practical debate about the purposes of child health systems and create space for new ideas about change processes and indicators of success to be developed. Otherwise, as we have seen, the aspirations of QI for genuine improvement are likely to undermined by being conflated with short-term institutional priorities.

'Good QI' is not of one type: there are good reasons, around cost, resourcing and expedience, to justify initiatives that improve things in the here and now rather than waiting for longer-term, more radical change. However, unless the potential for transformation is always in mind, we can never hope to move health systems from where they are to where they need to be. We encourage critical engagement with QI from health professionals to ensure that it embodies the rich conceptions of quality that motivate them and their patients. In addition, this means they will recognise when repair is valuable in its own right and also when it might be used as a stepping stone towards the goal of much-needed health system reform.

Correction notice This paper has been corrected since it was published online. The Wellcome Trust grant code acknowledged in the article has been updated.

\section{Twitter Guddi Singh @DrGuddiSingh}

Contributors GS and AC jointly conceived of the article and contributed to the writing of the manuscript.

Funding This work was supported by the Wellcome Trust (WT 209811_Z_17_Z)

Competing interests None declared.

Patient consent for publication Not required.

Provenance and peer review Not commissioned; externally peer reviewed. 


\section{Data availability statement No data are available.}

Open access This is an open access article distributed in accordance with the Creative Commons Attribution 4.0 Unported (CC BY 4.0) license, which permits others to copy, redistribute, remix, transform and build upon this work for any purpose, provided the original work is properly cited, a link to the licence is given, and indication of whether changes were made. See: https:// creativecommons.org/licenses/by/4.0/.

\section{ORCID iD}

Guddi Singh http://orcid.org/0000-0003-3263-393X

\section{REFERENCES}

1 Care Quality Commission. Quality improvement in hospital trusts: sharing learning from trusts on a journey of QI 2018.

2 Wolfe I, Cass H, Thompson MJ, et al. Improving child health services in the UK: insights from Europe and their implications for the NHS reforms. BMJ 2011;342:d1277.

3 Runnacles J, Moult B, Lachman P. Developing future clinical leaders for quality improvement: experience from a London children's hospital. BMJ Qual Saf 2013;22:956-63.

4 Doran NJ, Bethune R, Watson J, et al. Empowering junior doctors: a qualitative study of a QI programme in South West England. Postgrad Med J 2018;94:571-7.

5 Freed GL, deJong N, Macy ML, et al. Pediatricians' participation in quality improvement at the time of enrollment in MOC. Pediatrics 2018;142:e20180712.

6 Peterson A, Hanberger L, Akesson K, et al. Improved results in paediatric diabetes care using a quality registry in an improvement collaborative: a case study in Sweden. PLoS One 2014;9:e97875.

7 White CM, Statile AM, White DL, et al. Using quality improvement to optimise paediatric discharge efficiency. BMJ Qual Saf 2014;23:428-36.

8 Kerrey BT, Mittiga MR, Rinderknecht AS, et al. Reducing the incidence of oxyhaemoglobin desaturation during rapid sequence intubation in a paediatric emergency department. BMJ Qual Saf 2015;24:709-17.

9 Batalden P. Getting more health from healthcare: quality improvement must acknowledge patient coproduction-an essay by Paul Batalden. BMJ 2018;1:k3617.

10 Gardner J. Rethinking the clinical gaze: patient-centred innovation in paediatric neurology. Springer, 2017.

11 Boaden R, Harvey G, Moxham C, et al. Quality improvement: theory and practice in healthcare. Coventry, UK: NHS Institute for Innovation and Improvement/Manchester Business School, 2008.

12 Braithwaite J. Changing how we think about healthcare improvement. BMJ 2018;163:k2014.

13 Deming WE. The new economics for government, industry, education. Cambridge Mass: MIT Press, 1994.

14 Juran JM, De Feo J. Juran's Quality Handbook: The complete guide to performance excellence (sixth edition. New York, NY: McGraw Hill Education, 2010.

15 Reed JE, Davey N, Woodcock T. The foundations of quality improvement science. Future Hosp J 2016;3:199-202.

16 Hollnagel E, Wears RL, Braithwaite J. From Safety-I to Safety-II: a white paper. The resilient health care net: published simultaneously by the University of southern Denmark, University of Florida, USA, and. Australia: Macquarie University, 2015.

17 Ovretveit J. Learning about improvement to address global health and healthcare challenges-lessons and the future. Int J Qual Health Care 2018;30:37-41.
18 Dixon-Woods M. How to improve healthcare improvement-an essay by Mary Dixon-Woods. BMJ 2019;367:15514.

19 Junghans T. "Don't Mind the Gap!" Reflections on Improvement Science as a Paradigm. Health Care Anal 2018;26:124-39.

20 Dixon-Woods M, Martin GP. Does quality improvement improve quality? Future Hosp J 2016;3:191-4.

21 Carter SM. Valuing healthcare improvement: implicit norms, explicit normativity, and human agency. Health Care Anal 2018;26:189-205.

22 Smith K, Bambra C, Hill S. Background and introduction: UK experiences of health inequalities. Health inequalities, critical perspectives.; 2016: 1-21.

23 Feo R, Kitson A. Promoting patient-centred fundamental care in acute healthcare systems. Int J Nurs Stud 2016;57:1-11.

24 Checkland K. National service frameworks and UK general practitioners: street-level bureaucrats at work? Sociol Health Illn 2004;26:951-75.

25 Davies H, Powell A, Rushmer R. Why don't clinicians engage with quality improvement? 2007.

26 British Medical Association (2016). "Care Quality Commission inspection regime damaging frontline patient care, warns new BMA survey of 1,900 GP practices”. Press Release. Available: https:/www.bma.org.uk/news/media-centre/press-releases/ 2016/january/care-quality-commission-inspection-regimedamaging-frontline-patient-care

27 Cribb A. Improvement science meets improvement scholarship: Reframing research for better healthcare. Health Care Anal 2018;26:109-23.

28 Donabedian A. Evaluating the quality of medical care. Milbank Mem Fund Q 1966;44:166-206.

29 Plsek PE, Greenhalgh T. "The challenge of complexity in health care". Bmj 2001;323:625-8.

30 Bate R, Robert G, Fulop N, et al. Perspectives on context: a selection of essays. London: Health Foundation, 2014.

31 Liberati EG, Tarrant C, Willars J, et al. How to be a very safe maternity unit: an ethnographic study. Soc Sci Med 2019;223:64-72.

32 Allen D, Braithwaite J, Sandall J, et al. The sociology of healthcare safety and quality. Chichester, UK: Wiley-Blackwell, 2016.

33 Batalden M, Batalden P, Margolis P, et al. Coproduction of healthcare service. BMJ Qual Saf 2016;25:509-17.

34 Donetto S, Pierri P, Tsianakas V, et al. Experience-based codesign and healthcare improvement: realizing participatory design in the public sector. Int J Des 2015;18:227-48.

35 Valles SA. Philosophy of population health: philosophy for a new public health era. Routledge, 2018.

36 Wyatt R, Laderman M, Botwinick L, et al. Achieving health equity: a guide for Health Care Organizations. Cambridge, MA: Institute for Healthcare Improvement, 2016.

37 Boozary AS, Shojania KG. Pathology of poverty: the need for quality improvement efforts to address social determinants of health. BMJ Qual Saf 2018;27:421-4.

38 Leape LL, Shore MF, Dienstag JL, et al. Perspective: a culture of respect, part 1: the nature and causes of disrespectful behavior by physicians. Acad Med 2012;87:845-97.

39 Sokol-Hessner L, Folcarelli PH, Sands KEF. Emotional harm from disrespect: the neglected preventable harm. BMJ Qual Saf $2015 ; 24: 550-3$.

40 Jabbal J. Embedding a culture of quality improvement. London: The King's Fund, 2017. 
41 Andrews ET, Wootton S, Cable D, et al. Embedding electronic growth charts into clinical practice at a children's hospital. Arch Dis Child Educ Pract Ed 2018;103:82-4.

42 Weber EJ, Mason S, Carter A, et al. Emptying the corridors of shame: organizational lessons from England's 4-hour emergency throughput target. Ann Emerg Med 2011;57:79-88.

43 Mason S, Weber EJ, Coster J, et al. Time patients spend in the emergency department: England's 4-hour rule-a case of hitting the target but missing the point? Ann Emerg Med 2012;59:341-9.

44 NHS England. Clinically-led review of NHS access standards: interim report from the NHS national medical director, 2019. Available: https://www.england.nhs.uk/wp-content/uploads/ 2019/03/CRS-Interim-Report.pdf
45 Hannah S. Working upstream with QI to improve health and wellbeing outcomes - a national approach. Keynote presentation at the 2nd annual Child Health Festival at the Royal Society of Medicine. London, U.K, 2019.

46 Singh G, Zhu H. Poverty in practice: using quality improvement in paediatrics to improve identification and support of families living in poverty. Arch Dis Child Educ Pract Ed:edpract-2019-318259.

47 Padgett DK, Brodsky B. Psychosocial factors influencing nonurgent use of the emergency room: a review of the literature and recommendations for research and improved service delivery. Soc Sci Med 1992;35:1189-97. 